EPJ manuscript No.

(will be inserted by the editor)

\title{
Effect of a lattice upon an interacting system of electrons in two dimensions: Breakdown of scaling and decay of persistent currents
}

\author{
H. Falakshahi ${ }^{(a)}$, Z. Á. Németh ${ }^{(a, b)}$ and J.-L. Pichard ${ }^{(a, c)}$ \\ (a) CEA/DSM, Service de Physique de l'Etat Condensé, Centre d'Etudes de Saclay, 91191 Gif-sur-Yvette Cedex, France \\ (b) Eötvös University, Departement of Physics of Complex Systems, 1117 Budapest, Pázmány Péter sétány 1/A, Hungary \\ (c) Laboratoire de Physique Théorique et Modélisation, Universtité de Cergy-Pontoise, 95031, Cergy-Pontoise Cedex, France
}

\begin{abstract}
The ground state of an electron gas is characterized by the interparticle spacing to the effective Bohr radius ratio $r_{s}=a / a_{B}^{*}$. For polarized electrons on a two dimensional square lattice with Coulomb repulsion, we study the threshold value $r_{s}^{*}$ below which the lattice spacing $s$ becomes a relevant scale and $r_{s}$ ceases to be the scaling parameter. For systems of small ratios $s / a_{B}^{*}, s$ becomes only relevant at small $r_{s}$ (large densities) where one has a quantum fluid with a deformed Fermi surface. For systems of large $s / a_{B}^{*}, s$ plays also a role at large $r_{s}$ (small densities) where one has a Wigner solid, the lattice limiting its harmonic vibrations. The thermodynamic limit of physical systems of different $a_{B}^{*}$ is qualitatively discussed, before quantitatively studying the lattice effects occurring at large $r_{s}$. Using a few particle system, we compare exact numerical results obtained with a lattice and analytical perturbative expansions obtained in the continuum limit. Three criteria giving similar values for the lattice threshold $r_{s}^{*}$ are proposed. The first one is a delocalization criterion in the Fock basis of lattice site orbitals. The second one uses the persistent current which can depend on the interaction in a lattice, while it becomes independent of the interaction in the continuum limit. The third one takes into account the limit imposed by the lattice to the harmonic vibrations of the electron solid.
\end{abstract}

PACS. 71.10.-w Theories and models for many-electron systems - 71.10.Fd Lattice fermion models 73.20.Qt Electron solids

\section{Introduction}

When one considers interacting electrons free to move in an immobile background of positive ions, one can represent the ions by a uniform positive jellium if the electron density is sufficiently small. This uniform jellium gives simply rise to a constant term in the Hamiltonian. One gets a continuum model characterized by two scales: the inter-electron spacing $a$ and the effective Bohr radius $a_{B}^{*}$. Simple scaling laws are obtained if one uses the dimensionless ratio $r_{s}=a / a_{B}^{*}$. This continuum approximation neglects the discrete character of the lattice of positive ions.

If one wants to keep the periodic character of the ionic lattice, one has to include a periodic potential instead of a uniform jellium or to use the tight-binding approximation. One obtains a lattice model, where the kinetic energy can be simplified if the hopping terms are restricted to nearest neighbor ions. A lattice introduces a third scale: the lattice spacing $s$. If $s$ is irrelevant, the lattice model keeps the same universal scaling than the continuum limit, if one uses the combination of lattice parameters which becomes $r_{s}=a / a_{B}^{*}$ in the continuum.
We study when the low energy spectrum of the lattice model can be described by a continuum approximation, the lattice effects remaining only important in the high energy spectrum. What is the carrier density above which the scale $s$ becomes relevant and $r_{s}$ ceases to be the scaling parameter for the lattice ground state? The answer depends on $s$ and on the two parameters controlling the effective Bohr radius

$$
a_{B}^{*}=\frac{\varepsilon_{r} \hbar^{2}}{m^{*} e^{2}}:
$$

the effective mass $m^{*}$ of the carriers and the dielectric constant $\varepsilon_{r}$ of the medium.

If $a_{B}^{*}$ is large compared to $s$, the lattice effects are only important for small values of $r_{s}$, for which one has a quantum fluid with a deformed Fermi surface. This is a highly quantum weak coupling limit of large carrier densities. If the issue is to study charge crystallization in such systems, the densities of interest are much lower than those required to deform the Fermi surface, and the physics can be described in the continuum approximation. If one uses a $L \times L$ lattice model with $N$ particles to study electron crystallization for systems of small $s / a_{B}^{*}$, one has to take 
lattice fillings $N / L^{2}$ and hence ratios $r_{s}$ above the lattice threshold $r_{s}^{*}$, where the lattice model can be described by the continuum limit.

If $a_{B}^{*}$ is small compared to $s$, the lattice effects become important when $r_{s}$ is large also. This is a limit where the lattice physics takes place also in a weakly quantum strong coupling regime, at low densities. If one continues to increase the density in those systems, one can eventually reach the limit usually described by a Hubbard model near half filling, where the lattice can give rise to a Mott insulator if the interaction is large enough. In contrast to the case where $a_{B}^{*}$ is large compared to $s$, the continuum approximation cannot be assumed for studying electron crystallization. One has the obvious problem of commensurability between the electron lattice characterizing the continuum limit and the ionic lattice. Even if there is commensurability, there is a remaining limit imposed by the lattice to the harmonic vibrations of the Wigner solid.

Eventually, let us note that for a lattice model, it is important to know the electron density below which its low energy spectrum begins to exhibit the continuum behavior and its universal scaling laws, if one uses the combination of lattice parameters which becomes $r_{s}=a / a_{B}^{*}$ in the continuum.

\section{Two dimensional continuum model}

The Hamiltonian $H_{c}$ describing $N$ polarized electrons of mass $m$ free to move on a continuum space of dimension $d$ and dielectric constant $\varepsilon_{r}=1$ contains one body kinetic terms, two body interaction terms plus the constant term due to the presence of the uniform background of positive ions necessary to have charge neutrality.

$$
H_{c}=-\frac{\hbar^{2}}{2 m} \sum_{i=1}^{N} \nabla_{i}^{2}+e^{2} \sum_{1 \leq i<j \leq N} \frac{1}{\left|\mathbf{r}_{i}-\mathbf{r}_{j}\right|}+\text { const }
$$

Measuring the energies in rydbergs $\left(1 R y=m e^{4} / 2 \hbar^{2}\right)$ and the lengths in units of the radius $a$ of a sphere (circle in $2 d$ ) which encloses on the average one electron, $e$ and $m$ being the electronic charge and mass, $H_{c}$ becomes

$$
H_{c}=-\frac{1}{r_{s}^{2}} \sum_{i=1}^{N} \nabla_{i}^{2}+\frac{2}{r_{s}} \sum_{1 \leq i<j \leq N} \frac{1}{\left|\mathbf{r}_{i}-\mathbf{r}_{j}\right|}+\text { const }
$$

where $r_{s}=a / a_{B}$. The Bohr radius $a_{B}=\hbar^{2} / m e^{2}$ is a measure of the GS radius of the hydrogen atom while the rydberg $\left(1 R y=e^{2} /\left(2 a_{B}\right)\right)$ is its GS binding energy. Eq. 3 tells us that the physics of a system of interacting electrons in the continuum does not depend on many independent parameters $\left(\hbar, e, m\right.$, the electronic density $\left.n_{s} \ldots\right)$ but only on a single dimensionless scaling ratio $r_{s}=a / a_{B}$ when $N \rightarrow \infty, a_{B}$ characterizing the scale for the quantum effects.

For the GS, if many electrons are inside the quantum volume $a_{B}^{d}$, one gets the weak coupling limit (small $r_{s}$ ) where one has a Fermi liquid. Though our theory could be easily extended to arbitrary dimensions and could include the spin degrees of freedom, we restrict the study to polarized electrons (spinless fermions) and to the dimension $d=2$. The ground state (GS) energy in rydbergs per particle is given in the Hartree-Fock approximation [1] as:

$$
E_{0}=\frac{h_{0}}{r_{s}^{2}}+\frac{h_{1}}{r_{s}}+O\left(\ln r_{s}\right), \quad r_{s} \ll 1
$$

with coefficients $h_{0}=2$ for the kinetic energy and $h_{1}=$ -1.6972 for the exchange energy.

In the strong coupling limit (large $r_{s}$ ), the volume per electron $a^{d}$ is large compared to $a_{B}^{d}$, and the electrons crystallize on an hexagonal lattice with weak quantum effects (Wigner crystal). As Wigner's original approximation [2] suggests, the GS energy per particle in rydbergs can be expanded in powers of $r_{s}^{1 / 2}$ :

$$
E_{0}=\frac{f_{0}}{r_{s}}+\frac{f_{1}}{r_{s}^{3 / 2}}+\frac{f_{2}}{r_{s}^{2}}+O\left(r_{s}^{5 / 2}\right), \quad r_{s} \gg 1
$$

The leading term $\left(\propto r_{s}^{-1}\right)$ is of classical nature (Coulomb energy of the lattice of electrons in a continuum background of positive charge) while the first correction $(\propto$ $r_{s}^{-3 / 2}$ ) is quantum (zero point energy of the harmonic oscillations of the electrons about their lattice points). One gets [3] 4,5] $f_{0}=-2.2122$ and $f_{1}=1.628$ in two dimensions.

Using quantum Monte Carlo methods, the two dimensional crossover between these two limits has been studied. A variational approach [5] and a Green function Monte Carlo approach [6] have given a critical ratio $r_{s}^{c} \approx 37$ for a possible transition separating the quantum fluid from the Wigner solid in the continuum. However, the well-known sign problem of the Monte Carlo methods requires to impose the nodal structures of the solutions, making this picture not free of certain assumptions.

Assuming periodic boundary conditions (BCs) for $N$ polarized electrons in a square of size $D$, one can ignore the constant term in $H_{c}$, the electronic density $n_{s}=N / D^{2}$ and $a=1 / \sqrt{\pi n_{s}}$.

\section{Square lattice model}

We now define a square lattice model of spacing $s$, size $L=D / s$, nearest neighbor hopping element

$$
t=\frac{\hbar^{2}}{2 m s^{2}}
$$

and interaction strength

$$
U=\frac{e^{2}}{s}
$$

The lattice Hamiltonian $H_{l}$ reads:

$$
H_{l}=t\left(4 N-\sum_{\left\langle\mathbf{j}, \mathbf{j}^{\prime}\right\rangle} c_{\mathbf{j}}^{\dagger} c_{\mathbf{j}^{\prime}}\right)+\frac{U}{2} \sum_{\mathbf{j} \neq \mathbf{j}^{\prime}} \frac{n_{\mathbf{j}} n_{\mathbf{j}^{\prime}}}{\left|d_{\mathbf{j} \mathbf{j}^{\prime}}\right|} .
$$




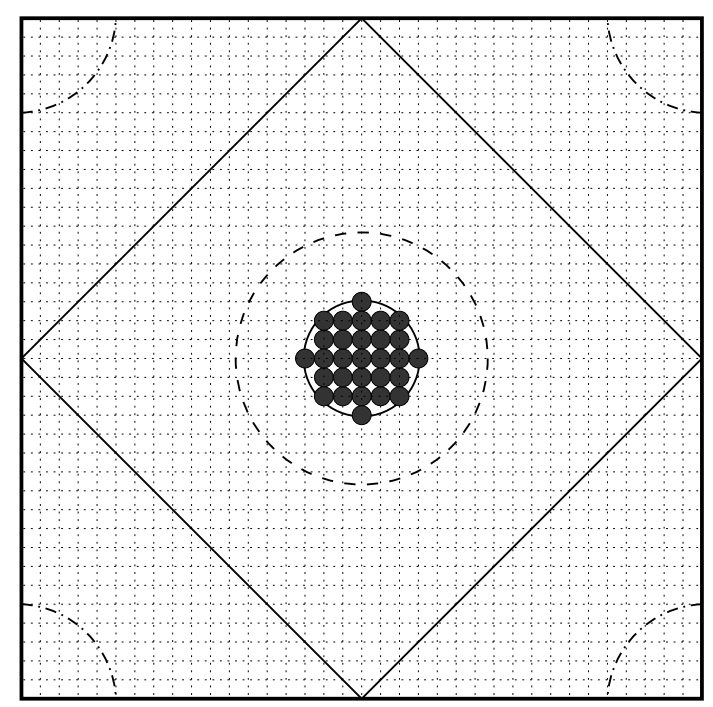

Fig. 1. Weak coupling limit: GS occupation numbers of a non interacting system in the reciprocal lattice. The Fermi-surfaces are sketched for increasing numbers of particles in a $36 \times 36$ square lattice. At low fillings, the Fermi surface is almost a circle, while it becomes deformed at larger fillings.

The operators $c_{\mathbf{j}}^{\dagger}\left(c_{\mathbf{j}}\right)$ create (annihilate) a polarized electron (spinless fermion) at the site $\mathbf{j}$ and $\left\langle\mathbf{j}, \mathbf{j}^{\prime}\right\rangle$ means that the sum is restricted to nearest neighbors. $d_{\mathrm{ji}^{\prime}}$ is the distance between the sites $\mathbf{j}$ and $\mathbf{j}^{\prime}$ in unit of $s$.

The Hamiltonian (8) can also be written using the operators $d_{\boldsymbol{k}}^{\dagger}\left(d_{\boldsymbol{k}}\right)$ creating (annihilating) a polarized electron in a plane wave state of momentum $\boldsymbol{k}$ :

$$
\begin{aligned}
H_{l}= & 4 N t-2 t \sum_{k}\left(\cos k_{x}+\cos k_{y}\right) d_{\mathbf{k}}^{\dagger} d_{\mathbf{k}} \\
& +U \sum_{\mathbf{k}, \mathbf{k}^{\prime}, \mathbf{q}} V(\mathbf{q}) d_{\mathbf{k}+\mathbf{q}}^{\dagger} d_{\mathbf{k}^{\prime}-\mathbf{q}}^{\dagger} d_{\mathbf{k}^{\prime}} d_{\mathbf{k}}
\end{aligned}
$$

where

$$
V(\mathbf{q})=\frac{1}{2 L^{2}} \sum_{\mathbf{j}} \frac{\cos \mathbf{q} \mathbf{j}}{d_{\mathbf{j} \mathbf{0}}} .
$$

The states of different total momenta $\boldsymbol{K}$ are decoupled. In the lattice units, $1 R y=U^{2} / 4 t, a_{B}=2 s t / U$ and the ratio $r_{s}$ becomes:

$$
r_{s}=\frac{a}{a_{B}}=\frac{U L}{2 t \sqrt{\pi N}}
$$

\section{Lattice effects in the thermodynamic limit}

\subsection{Lattice threshold in the weak coupling limit}

In the limit $r_{s} \rightarrow 0$, the GS energy is mainly kinetic. This is a consequence of the Pauli exclusion principle, which pushes the Fermi energy of the non interacting system

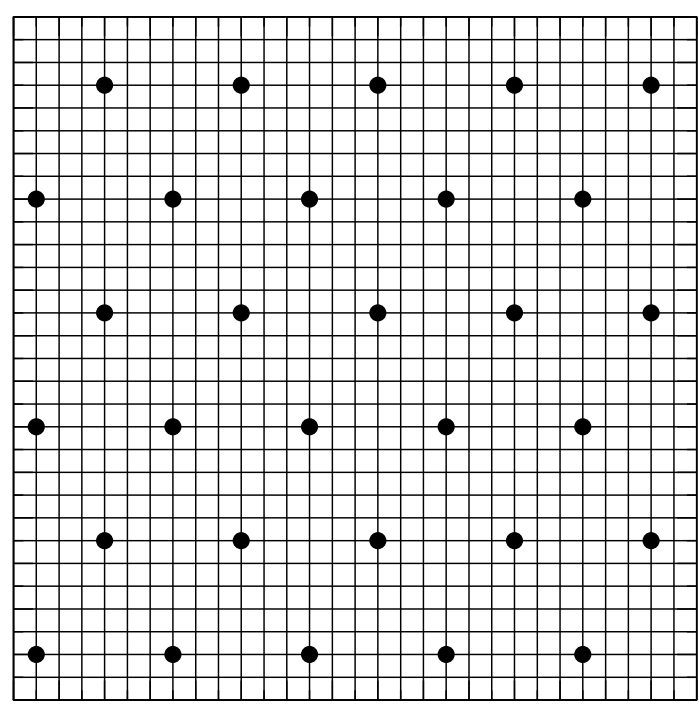

Fig. 2. Strong coupling limit: GS occupation numbers of a Wigner solid in real space. The hexagonal Wigner lattice of $N$ electrons becomes commensurate with the $L \times L$ square lattice if one takes $N=L=30$.

to much higher values than the classical Coulomb energy. The GS kinetic energies of $H_{l}$ and $H_{c}$ become different when the Fermi surface is deformed by the lattice. Such a deformation can give rise to nesting effects with important consequences discussed in the literature $[7,8,9$. The Fermi wave vectors $\mathbf{k}_{\mathbf{F}}$ are given by $4 t-2 t\left(\cos k_{x}^{F}+\cos k_{y}^{F}\right)=\epsilon_{F}$ for a square lattice of Fermi level $\epsilon_{F}$, instead of $t\left(k_{x}^{F}+\right.$ $\left.k_{y}^{F}\right)^{2}=\epsilon_{F}$ for the continuum limit.

Expanding the kinetic part of the lattice Hamiltonian (9) for small wave vectors, one gets:

$$
H_{l}^{k i n} \approx t \sum_{k<k_{F}} \mathbf{k}^{2}-\frac{t}{12} \sum_{k<k_{F}}\left(k_{x}^{4}+k_{y}^{4}\right)
$$

For the first term, one recovers the kinetic term of the continuum expansion (4):

$$
\begin{aligned}
t \sum_{k<k_{F}} \mathbf{k}^{2} & \approx \int \frac{D^{2}}{(2 \pi)^{2}} \frac{\hbar^{2}}{2 m} \mathbf{k}^{2} d^{2} \mathbf{k} \\
& =\frac{N^{2} \hbar^{2} \pi}{m D^{2}}=\frac{2}{r_{s}^{2}}(N R y),
\end{aligned}
$$

while the lattice correction reads:

$$
\begin{aligned}
\Delta E_{l} & =-\frac{t}{12} \sum_{\mathbf{k}} k_{x}^{4}+k_{y}^{4} \\
& =-\frac{\hbar^{2}}{24 m s^{2}} \int \frac{L^{2}}{(2 \pi)^{2}}\left(k_{x}^{4}+k_{y}^{4}\right) d^{2} \mathbf{k}
\end{aligned}
$$

which becomes, using $k_{F}=\sqrt{4 N \pi} / L$ :

$$
\Delta E_{l}=-\frac{\hbar^{2} N^{3} \pi^{2}}{m L^{4} s^{2}}=-N R y \frac{2}{r_{s}^{2}} \frac{\pi N}{L^{2}} .
$$


The condition for having the lattice correction $\Delta E_{l}$ (Eq. 15) smaller than the continuum kinetic energy (Eq. [13) yields

$$
r_{s}>r_{s}^{*} \approx \frac{s}{a_{B}}
$$

This estimate of $r_{s}^{*}$ is only valid when $r_{s} \rightarrow 0$, since it neglects the effect of the interaction. When one turns on the interaction, transitions from states below the Fermi surface to states above it (see Hamiltonian (9) and Fig. 11) take place. This smears the Fermi surface, giving an uncertainty $\Delta k_{F}$ to $k_{F}$. This uncertainty is evaluated in Appendix A One gets:

$$
\Delta k_{F}=\frac{U^{2} L}{t^{2} \sqrt{N}} \frac{I^{2}}{16 \sqrt{4 \pi}} .
$$

Since $\cos \left(k_{F}+\Delta k_{F}\right) \approx 1-\left(k_{F}+\Delta k_{F}\right)^{2} / 2$ when $k_{F}+\Delta k_{F}$ is small (say $k_{F}+\Delta k<\pi / 2$ ), one finds that the previous estimate $\propto s / a_{B}$ for $r_{s}^{*}$ is increased by an interaction effect $\propto\left(s / a_{B}\right)^{3}$ for small $r_{s}$.

\subsection{Lattice threshold in the strong coupling limit}

In the strong coupling limit $r_{s} \rightarrow \infty$, the GS energy is mainly classical (Coulomb energy) with weak quantum corrections. The electron lattice minimizing the Coulomb energy can be different if the square lattice of the model is not commensurate with the hexagonal lattice that the electrons form in the continuum limit. This is one obvious source of lattice effects when $r_{s} \rightarrow \infty$. One does not discuss it, restricting the study to values of $N$ and $L$ where the two lattices are commensurate and give the same Coulomb energy. In this case, the lattice can nevertheless change the vibration modes of the electron system around its classical electrostatic limit, when $\hbar \rightarrow 0$. Let us consider the leading quantum corrections to the classical energy.

In the continuum model, the GS energy per particle in rydbergs is given by Eq. [5] The first quantum correction $\Delta E_{0}^{c}\left(r_{s} \rightarrow \infty\right)$ to the Coulomb energy $\left(\propto r_{s}^{-1}\right)$ is given by the zero point energy of the harmonic oscillations of the electrons around their lattice points:

$$
\Delta E_{0}^{c}\left(r_{s} \rightarrow \infty\right)=\frac{f_{1}}{r_{s}^{3 / 2}}
$$

In the lattice model, the classical limit $t \rightarrow 0$ is not described by the expansion in powers of $r_{s}^{1 / 2}$ valid in the continuum limit (Eq. 51), but by a lattice perturbation theory where the small parameter is $t^{2} / U$. Examples of this lattice expansion valid when $t^{2} / U \rightarrow 0$ can be found in refs. 10,11,12,13,14. Its dominant quantum correction $\Delta E_{0}^{l}\left(r_{s} \rightarrow \infty\right)$ to the classical Coulomb energy comes from the term $4 N t$ of the lattice Hamiltonian (8). Expressed in rydbergs $\left(1 R y=U^{2} / 4 t\right)$ per particle, this gives

$$
\Delta E_{0}^{l}\left(r_{s} \rightarrow \infty\right)=\frac{16 t^{2}}{U^{2}}
$$

\begin{tabular}{|c||c|c|c|c|c|}
\hline & $m^{*} / m_{0}$ & $\varepsilon_{r}$ & $a_{B}^{*}(\AA)$ & $\mathrm{s}(\AA)$ & $s / a_{B}^{*}$ \\
\hline \hline$(1)$ & 0.19 & 12 & 33,2 & 2.35 & 0.071 \\
\hline$(2)$ & 0.07 & 13 & 100,0 & 4.0 & 0.04 \\
\hline$(3)$ & 0.6 & 13 & 12 & 4.0 & 0.33 \\
\hline$(4)$ & 10 & 10 & 0.53 & 3.8 & 7.16 \\
\hline$(5)$ & 175 & 20 & 0.061 & 2.85 & 46.7 \\
\hline
\end{tabular}

Table 1. Typical physical parameters for two dimensional systems of charges of increasing effective masses created in various devices: (1) Si-Mosfet, (2) n-doped GaAs - GaAlAs heterostructure, (3) p-doped GaAs - GaAlAs heterostructure, (4) cuprate oxides exhibiting high- $T_{c}$ superconductivity and (5) layered sodium cobalt oxides $\mathrm{Na}_{x} \mathrm{CoO}_{2}$.

The next quantum corrections of order $t^{2} / U$ become negligible in the limit $t \propto \hbar^{2} \rightarrow 0$.

Let us consider a low density of electrons in a very large lattice, where one has the same hexagonal lattice with the same harmonic vibrations than in the continuum. The quantum corrections to the Coulomb energy and $r_{s}$ are then given by Eq. [18] and Eq. [1] respectively. If one takes the classical limit $\hbar \rightarrow 0$ in such a system, $\Delta E_{0}^{c}\left(r_{s}\right)$ will reach the lattice limit $\Delta E_{0}^{l}\left(r_{s}\right)$ which cannot be exceeded. This corresponds to coupling strengths where the harmonic vibrations of the electron lattice become so small that the discrete nature of the available space [12,13] becomes relevant. The lattice threshold $r_{s}^{*}$ is then obtained from the condition $\Delta E_{0}^{c}\left(r_{s}\right) \approx \Delta E_{0}^{l}\left(r_{s}\right)$ and the continuum approximation is only valid if:

$$
\frac{N}{L^{2}}<\frac{1}{\pi}\left(\frac{4^{5}}{f_{1}^{4}}\right)^{1 / 3}\left(\frac{t}{U}\right)^{2 / 3} .
$$

Using the lattice spacing to the Bohr radius ratio $s / a_{B}$, one gets that the lattice GS can be described by a continuum theory in the thermodynamic limit if

$$
r_{s}>r_{s}^{*}=0.55\left(\frac{s}{a_{B}}\right)^{4 / 3}
$$

and exhibits lattice effects otherwise.

\subsection{Two dimensional systems of different $a_{B}^{*}$}

If the effective mass of the carriers is $m^{*}$ in a medium of dielectric constant $\varepsilon_{r}$, one must replace $a_{B}$ by the corresponding effective Bohr radius $a_{B}^{*}$. Some typical values of $m^{*} / m_{0}, \varepsilon_{r}, a_{B}^{*}, s$ and $s / a_{B}^{*}$ are given in Table 1 for two dimensional systems of charges created in various systems. In general, the lattice spacing $s$ is always of a few angstroms, and $\varepsilon_{r} \approx 10$. But the carriers can be light or heavy, as indicated in Table 1

When they are light, as in Ga-As heterostructures or in Si-Mosfets, $s$ is small compared to $a_{B}^{*}$, and $r_{s}^{*}$ is small. The immobile ions can be modeled by a continuum uniform jellium, unless one reaches the very large densities where the Fermi surface becomes deformed. These densities are out of reach in today's semi-conductor field effect devices. 
Therefore, if one numerically studies charge crystallization for those systems using a lattice model, $U / t, L$ and $N$ must be taken such that $r_{s}=(U L / 2 t) /(\sqrt{\pi N})>r_{s}^{*}$.

In the cuprate oxides exhibiting high- $T_{c}$ superconductivity, the effective mass of the carriers is heavier, though not large enough for having $s / a_{B}^{*}$ above 23 , the value for which $0.55\left(s / a_{B}^{*}\right)^{4 / 3} \approx 37$. The deformation of the Fermi surface by the lattice yields nesting effects which can be responsible for singlet d-wave superconductivity [15] in those oxides. The lattice filling is large, reaching the limit where one can take a Hubbard model near halffilling. When one goes away from half-filling by chemical doping, those systems can reach a more dilute limit where one gets a quantum fluid which can be described by a continuum theory and where the scaling parameter $r_{s}$ becomes relevant.

There are systems with much larger effective masses, as those described by heavy fermions theories where $s / a_{B}^{*}$ can exceed 23. One example is given [16 by layered Lithium or Sodium Cobalt oxides: $\mathrm{Li}_{x} \mathrm{CoO}_{2}$ or $\mathrm{Na}_{x} \mathrm{CoO}_{2}$, where the effective mass of carriers can reach $\approx 200$, a value more familiar to $f$-band heavy fermions than to $d$-band metals, as discussed by Roger and Shannon[17. Those systems look particularly interesting for the subject of this study, since one should observe by increasing the carrier concentration a continuum-lattice transition for the electron (or hole) crystal, before having the quantum melting of this crystal in the lattice solid regime, to eventually obtain at higher densities a quantum fluid with a deformed Fermi surface.

In Fig. 3 the regime of validity of the continuum approximation is given in the $\left(s / a_{B}^{*}, a / a_{B}^{*}\right)$ plane, for typical Ga-As heterostructures, cuprate oxides or layered sodium cobalt oxides $\mathrm{Na}_{x} \mathrm{CoO}_{2}$. The dashed line $\nu=N / L^{2}=1$ corresponds to systems usually described by the half-filled Hubbard model, when the spins are included. Increasing the density, one goes towards a lattice regime where the continuum approximation breaks down, giving rise to a lattice liquid $\left(s / a_{B}^{*}<23\right)$ or to a lattice solid $\left(s / a_{B}^{*}>23\right)$.

\section{Scaling in a lattice model with a fixed number $N$ of particles}

Let us define the lattice parameter suitable when $N$ is constant.

$$
r_{l}=\frac{U L}{t}=r_{s}(2 \sqrt{\pi N})
$$

So far, we have considered the realistic case where $N$ is varied by an electrostatic gate or by chemical doping in a lattice where $L, t$ and $U$, and hence $r_{l}$ are given. One can also study the lattice-continuum crossover by varying the lattice parameter $r_{l}$ in a system of $N$ particles. If $N$ remains constant in a lattice where one varies $r_{l} \propto r_{s}$, one gets the continuum limit and its universal scaling for small values of $r_{l}$ while the lattice becomes relevant and the continuum scaling breaks down for large $r_{l}$.

This can be seen from Hamiltonian (9), where the components of the two dimensional vectors $\mathbf{k}, \mathbf{k}^{\prime}$ and $\mathbf{q}$ can

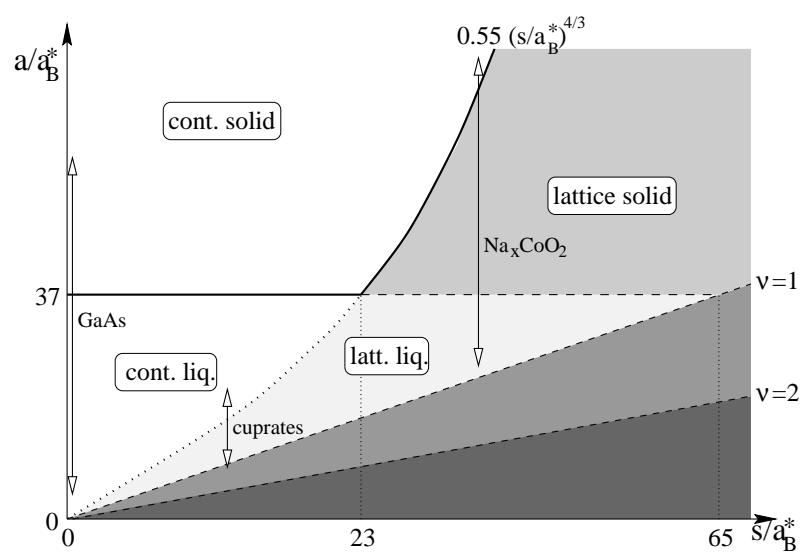

Fig. 3. Sketch of the lattice and continuum regimes in the $\left(s / a_{B}^{*}, a / a_{B}^{*}\right)$ plane. In the non-shaded part, the lattice GS can be described by a continuum theory. The shaded part below the dashed line $\nu=N / L^{2}=1$ (2 with spins) is forbidden in a lattice model (more than 1 ( 2 with spins) electron per site). The line $r_{s}=a / a_{B}^{*} \approx 37$ gives the density under which Wigner crystallization is assumed to occur in the continuum. The thick line $0.55\left(s / a_{B}^{*}\right)^{4 / 3}$ gives the lattice threshold $r_{s}^{*}$ above $r_{s} \approx 37$, while the dotted line gives $r_{s}^{*} \propto s / a_{B}^{*}+O\left(s / a_{B}^{*}\right)^{3}$ below $r_{s} \approx 37$. The three double arrows correspond to typical Ga-As heterostructures, cuprate oxides and layered sodium cobalt oxides $\mathrm{Na}_{x} \mathrm{CoO}_{2}$. Increasing the density, one goes from a continuum liquid or solid towards lattice regimes.

take the values $0,2 \pi / L, \ldots, 2 \pi(L-1) / L$. If the Fermi energy is sufficiently small for having $4-2\left(\cos k_{x}+\cos k_{y}\right) \approx$ $\mathbf{k}^{2}$ for all the states below the Fermi surface, the kinetic energy reads

$$
4 N t-2 t \sum_{\mathbf{k}<\mathbf{k}_{F}}\left(\cos k_{i x}+\cos k_{i y}\right) \approx \frac{4 \pi^{2} t}{L^{2}} \sum_{i=1}^{N} \mathbf{p}_{i}^{2},
$$

where $\mathbf{k}_{i}=(2 \pi / L) \mathbf{p}_{i}$ with $p_{x}, p_{y}<<L$ for all $\mathbf{k}_{i}<\mathbf{k}_{F}$. Expressed in rydbergs $\left(1 R y=U^{2} / 4 t\right)$, the diagonal matrix elements of Hamiltonian (9) due to the kinetic energy depend only on the lattice parameter $r_{l}$ :

$$
\frac{4 \pi^{2} t}{L^{2}} \sum_{i=1}^{N} \mathbf{p}_{i}^{2}=\frac{16 \pi^{2}}{r_{l}^{2}}\left(\sum_{i=1}^{N} \mathbf{p}_{i}^{2}\right) R y .
$$

The diagonal Coulomb matrix elements are given by $N(N-1) U V(\mathbf{q}=0)-\sum_{i \neq i^{\prime}} U V\left(\mathbf{k}_{i}-\mathbf{k}_{i^{\prime}}\right)$, while the offdiagonal terms $\propto U\left(V\left(\mathbf{k}_{i_{1}}-\mathbf{k}_{i_{1}^{\prime}}^{\prime}\right)-V\left(\mathbf{k}_{i_{1}}-\mathbf{k}_{i_{2}^{\prime}}^{\prime}\right)\right)$, where $V(\mathbf{q})$ is given by Eq.10] If $U$ is small, only the off-diagonal terms with small momentum transfers $(\mathbf{q}=(2 \pi / L) \mathbf{p}$ with $\left.p_{x}, p_{y}<<L\right)$ play a role. Transforming the discrete sum to a continuum integral, one gets for the interaction matrix elements:

$$
\begin{aligned}
U V(\mathbf{q}) & =\frac{U}{2 L^{2}} \sum_{\mathbf{j}} \frac{e^{i \mathbf{q} \mathbf{j}}}{d_{\mathbf{j}, 0}} \\
& =\frac{U}{2 L} \sum_{\mathbf{j}} \frac{e^{i L \mathbf{q} \frac{j}{L}}}{d_{\mathbf{j}, 0} / L} \frac{\Delta j_{x}}{L} \frac{\Delta j_{y}}{L}
\end{aligned}
$$




$$
\approx \frac{U}{2 L} \int_{0}^{1} \int_{0}^{1} \frac{e^{2 \pi i \mathbf{p r}}}{d(\mathbf{r}, 0)} d^{2} \mathbf{r}=\frac{U}{2 L} I(\mathbf{p}) .
$$

The integral $I(\mathbf{p})$ is independent 12 of $L$ when $p_{x}, p_{y}<<$ $L$ and the Coulomb matrix elements of Hamiltonian (9) become also a function of the lattice parameter $r_{l}$ only:

$$
U V(\mathbf{q})=\frac{4 t}{U^{2}} \frac{U}{2 L} I(\mathbf{p}) R y=\frac{2 I(\mathbf{p})}{r_{l}} R y .
$$

For $N$ fixed, assuming that $N$ is small enough for avoiding deformed Fermi surfaces without interaction, the low energy levels depend only on the lattice parameter $r_{l}$ when $r_{l}$ is small. The question is to determine the interaction threshold $r_{l}^{*}$ above which the off-diagonal interaction terms begin to delocalize this GS to states of higher momenta, where $4-2\left(\cos k_{x}+\cos k_{y}\right) \neq \mathbf{k}^{2}$. When $r_{l}$ exceeds this $r_{l}^{*}$, the lattice GS ceases to be a function of $r_{l} \propto r_{s}$ as in the continuum limit.

\section{Lattice effects for a few correlated particles}

We propose three criteria giving similar lattice thresholds $r_{l}^{*}$ for an interacting system of $N$ polarized electrons, which will be carefully studied when $N=3$ in the next section. The first one is a delocalization criterion in the Fock basis of lattice site orbitals. The second one uses the invariance of the persistent current when one varies the interaction strength in the continuum, an invariance which can be broken by the lattice. The third one is based on the limit imposed by the lattice to the zero point energy of the harmonic vibrations of an $N$ electron solid, as previously discussed in the thermodynamic limit.

\subsection{Criterion 1: Delocalization in the Fock basis of lattice site orbitals}

Let us consider the system of $N$ particles in real space instead of reciprocal space, in the limit $t=0$ where the $N$ electrons are localized on $N$ sites (see Fig. 2) and form states $|J\rangle=c_{\mathbf{j}_{1}}^{\dagger} \ldots c_{\mathbf{j}_{\mathbf{N}}}^{\dagger}|0\rangle$ of energy $E_{\text {Coul }}(J)$. As one turns on $t$, one can expect that the lattice becomes irrelevant as each electron ceases to be localized on a single site. In analogy with the problem of a single particle in a disordered lattice, one can use the criterion first proposed by Anderson 18: delocalization takes place when the hopping term $t$ between directly coupled sites becomes of the order of their energy spacing $\Delta E$. This criterion was extended to interacting systems in many different contexts: onset of quantum chaos in many body spectra [10,19, 20] and in the quantum computer core [21, quasiparticle lifetime and delocalization in Fock space [22,23]. In our case, the states become delocalized in the many body basis built from the states $|J\rangle$ when the matrix element $\left\langle J^{\prime}\left|H_{k i n}\right| J\right\rangle$ of the one body perturbation $H_{k i n} \propto t$ coupling a state $|J\rangle$ to the "first generation" of states $\left|J^{\prime}\right\rangle$ directly coupled to it by $H_{k i n}$ exceeds their energy spacing $\Delta E_{\text {Coul }}=E_{\text {Coul }}\left(J^{\prime}\right)-E_{\text {Coul }}(J)$. This gives $t>\Delta E_{\text {Coul }}$.
Applying this criterion to the GS, one obtains $r_{l}^{*}$ from the condition

$$
t \approx \Delta E_{\text {Coul }}
$$

where $\Delta E_{\text {Coul }}$ is the increase of Coulomb energy yielded by the hop of one particle localized on the GS configuration to a nearest neighbor site when $t=0$. When $t$ exceeds $\Delta E_{C o u l}$, the GS is delocalized on the $J$-basis, and hence on the lattice, and the lattice GS behaves as the continuum GS.

\subsection{Criterion 2: Persistent currents}

Since a continuum model is invariant under translations, the motion of the center of mass can be decoupled from the relative motions. Thus the continuum Hamiltonian $H_{c}$ (Eq. 3) can be decomposed in two parts, one related to the center of mass motion which is independent of the interaction, while the second one contains only the relative motions and hence the interaction. This has a very important consequence for the persistent current $I$ driven by an enclosed Aharonov-Bohm flux $\Phi$ in a continuum model: $I$ is independent of $r_{l}$ and keeps its non interacting value. For having the topology of a $2 d$ torus enclosing $\phi$ along the $x$-direction, one takes the corresponding curled $\mathrm{BC}$ in this direction, keeping periodic BC in the $y$-direction. For a sufficient $r_{l}$, the electrons form a Wigner solid and the small relative motions cannot feel the BCs. In this limit, $I$ is just given by the center of mass motion, which is independent of $r_{l}$, and hence coincides with its non-interacting value. This point remains correct for small $r_{l}$, as it was proven for $1 d$-rings [24,25,26] and observed for $d=2$ [24. In contrast, since the previous decomposition into two parts does not necessary hold for $H_{l}, I \neq I\left(r_{l}=0\right)$ for a lattice when $H_{l}$ and $H_{c}$ have different GSs. The decay of $I$ above $r_{l}^{*}$ (small $t / U$ at fixed $N$ and $L$ ) can be evaluated 11,12,14 by the leading contribution (of order $N) I_{l}^{(N)} \propto t(t / U)^{N-1}$ of the $t / U$ lattice expansion. The value of $r_{l}$ for which

$$
I(U=0) \approx I_{l}^{(N)}
$$

gives the Criterion 2 for $r_{l}^{*}$ (see Fig. 2 right). Instead of $I(\Phi)$, one can prefer to use the Kohn curvature $C_{K}=$ $\partial^{2} E_{0} / \partial \Phi^{2}$ evaluated at $\Phi=0$ or the GS energy change $\Delta E_{0}=E_{0}(\Phi=0)-E_{0}\left(\Phi=\Phi_{0} / 2\right)$ where $\Phi_{0}$ is the flux quantum. To apply $\Phi_{0} / 2$ corresponds to have anti-periodic $\mathrm{BC}$ in the $x$-direction.

\subsection{Criterion 3: Lattice limit for the zero point motion of an electron solid}

This is the criterion that we have already used in the qualitative discussion of the thermodynamic limit, and which we consider when $N$ is finite. When $t / U \rightarrow 0$, the leading correction to the Coulomb energy of $H_{l}$ is $4 N t$. Since the correction $E_{v i b}\left(r_{l}\right)$ to the Coulomb energy coming from the zero point vibrational motion of the continuum solid 
cannot exceed this lattice limit $4 N t, r_{l}^{*}$ can be obtained from the condition

$$
E_{v i b}\left(r_{l}^{*}\right) \approx 4 N t,
$$

assuming that the values of the lattice parameters can yield a Wigner solid for $r_{l}<r_{l}^{*}$.

\section{Numerical study of three polarized electrons}

When one takes periodic BCs, a convention has to be chosen for the distance $r$ (Hamiltonian (31) $)$ or $d_{\mathbf{j j}^{\prime}}$ (Hamiltonian (8)). For a finite square with periodic BCs, one possible definition is given by:

$$
d_{\mathbf{j j}^{\prime}}^{P S C}=\sqrt{\min \left(\left|d_{x}, L-\right| d_{x} \mid\right)^{2}+\min \left(\left|d_{y}\right|, L-\left|d_{y}\right|\right)^{2}},
$$

where $d_{x}=j_{x}-j_{x}^{\prime}$ and $d_{y}=j_{y}-j_{y}^{\prime}$. Hereafter, we refer to the corresponding $1 /\left|d_{\mathrm{jj}^{\prime}}\right|$ repulsion as the periodic singular Coulomb (PSC) repulsion, since it has a cusp when the interparticle distance $d_{\mathbf{j j}^{\prime}}$ has one of its coordinates equal to $L / 2$. This cusp being unphysical, we introduce the periodic regularized Coulomb (PRC) repulsion, defined from

$$
d_{\mathbf{j} \mathbf{j}^{\prime}}^{P R C}=\frac{L}{\pi} \sqrt{\sin ^{2} \frac{\left|d_{x}\right| \pi}{L}+\sin ^{2} \frac{\left|d_{y}\right| \pi}{L}}
$$

which locally coincides with the PSC repulsion, but remains analytic for all values of $d_{\mathbf{j} \mathbf{j}^{\prime}}$ when $s \rightarrow 0$. The PRC repulsion is essentially equivalent to the Ewald repulsion obtained from the periodic repetition of the considered system.

Defining $d_{\mathrm{jj}^{\prime}}$ with Eq. 31 we calculate the quantities used in the different criteria for $N=3$ polarized electrons on a square lattice. We give in Appendix $\mathrm{C}$ the same analysis defining $d_{\mathbf{j} j^{\prime}}$ with Eq. [30]instead of Eq. 31] The choice of the PRC or PSC repulsions, or of the repulsion obtained after Ewald summation is arbitrary for three particles in a toroidal geometry. Nevertheless, it allows us to check if our three proposed criteria for $r_{l}^{*}$ give consistent results when the long range form of the Coulomb repulsion is changed. The presented results extend to larger $L$ previous studies of the case $N=2$ and $N=3$ given in Ref. 13 and Ref.12 respectively.

For $t=0$, the configuration of particles minimizing the PRC Coulomb energies is given in the inset of Fig. 4. The values of $L=6,9,12,15,18, \ldots$ yield a diagonal Wigner molecule shown in the inset which is commensurate with the square lattice. Moving one particle by a single hop increases the Coulomb energy $E_{0}=(\sqrt{6} U \pi) / L$ by an amount

$$
\Delta E_{\text {Coul }} \approx \frac{7 \sqrt{2} \pi^{3} U}{12 \sqrt{3} L^{3}}
$$

when $L$ is sufficiently large.

For $U=0$, the GS energy is given by $E_{0}(0)=12 t-8 t-$ $4 t \cos (2 \pi / L)$ for periodic BCs and becomes $E_{0}\left(\Phi_{0} / 2\right)=$

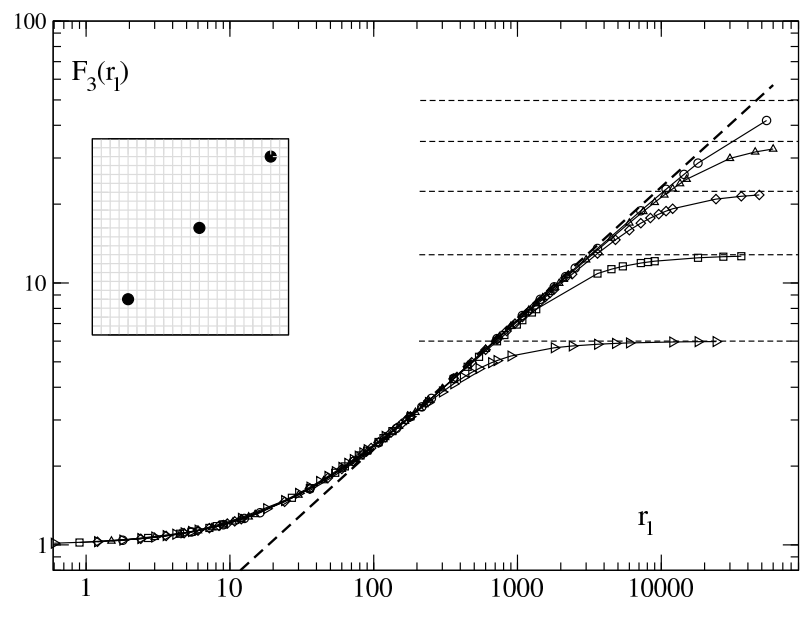

Fig. 4. Energy ratio $F_{N=3}(L, U, t)$ as a function of $r_{l}=U L / t$ given by the PRC repulsion for $L=6(\triangleright), 9(\square), 12(\diamond), 15(\triangle)$, 18 (०). The dotted-dashed line gives the behavior $0.2327 \sqrt{r_{l}}$ (harmonic vibrations of the continuum Wigner molecule) and intersects the limiting dashed lines $12 t /(4 t-4 t \cos (2 \pi / L))$ at the $r_{l}^{*}(L)$ corresponding to criterion 3. Inset: A GS configuration when $t=0$ and $L=24$.

$12 t-8 t \cos (\pi / L)-4 t \cos (3 \pi / L)$ when one twists the $\mathrm{BC}$ in the $x$-direction. The difference $\Delta E_{0}=E_{0}\left(\Phi_{0} / 2\right)-E_{0}(0) \approx$ $-14 \pi^{2} t / L^{2}$ when $L \rightarrow \infty$. When $t / U$ is small, $\Delta E_{0}$ can be calculated at the leading order of a $t / U$-expansion [12] for $N=3$. This gives when $L$ is large:

$$
\lim _{r_{l} \rightarrow 0} \Delta E_{0} \approx \frac{14 \pi^{2} t}{L^{2}} ; \lim _{r_{l} \rightarrow \infty} \Delta E_{0} \approx \frac{9 \pi^{2} t^{3}}{L^{2} \Delta E_{\text {Coul }}}
$$

where $\Delta E_{\text {Coul }}$ is given by the Eq. 32 Using these expressions, one obtains from the two first criteria:

$$
r_{l}^{*}(L)=A L^{\alpha}
$$

where $\alpha=4$ for the PRC repulsion, the constant $A$ slightly depending on the taken criterion.

\subsection{Persistent currents}

We now present numerical results obtained using the Lanczos algorithm, using Hamiltonian (9) and considering the sub-space of total momentum $\mathbf{K}=0$ [12] for periodic BCs (no applied flux).

Our system has the topology of a $2 d$ torus. To enclose an Aharonov-Bohm flux $\Phi$ along the $x$-direction, one takes the corresponding curled $\mathrm{BC}$ in this direction while the $\mathrm{BC}$ in the $y$-direction remains periodic. To apply half a flux quantum $\left(\Phi=\Phi_{0} / 2\right)$ is equivalent to take antiperiodic $\mathrm{BC}$ along the $x$-direction. In Fig $[5$ the increase $E_{0}(\Phi)-E_{0}(0)$ of the GS energy $E_{0}$ is given as a function of $\Phi / \Phi_{0}$ for different values of $r_{l}$ using a $18 \times 18$ square lattice. When $r_{l}$ is small, the curves coincide. This is the continuum regime where the persistent current is 


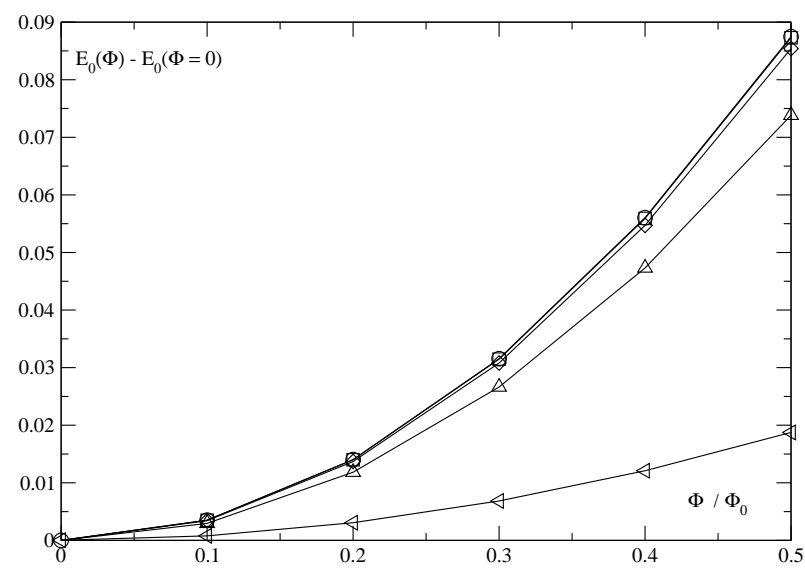

Fig. 5. GS energy $E_{0}(\Phi)-E_{0}(\Phi=0)$ as a function of the enclosed dimensionless magnetic flux $\Phi / \Phi_{0}$ for $N=3, L=18$, PRC interaction at $r_{l}=6(\circ), 60(\square), 600(\diamond) 6000(\triangle)$ and $60000(\triangleleft)$.

independent of the interaction. When $r_{l}$ is large, the increase $E_{0}(\Phi)-E_{0}(0)$ becomes weaker. This is the lattice regime where the persistent current decays as the interaction increases. One gives in Fig 6 the dimensionless change $\Delta E_{0}\left(r_{l}\right) / \Delta E_{0}\left(r_{l}=0\right)$ of the GS energy when the $\mathrm{BC}$ is twisted in the $x$-direction for increasing values of $L$. One can see the two limits given by Eq. 33 , $\Delta E_{0}\left(r_{l}\right) / \Delta E_{0}\left(r_{l}=0\right) \approx 1$ in the continuum limit, followed by a decay when $r_{l}$ exceeds the lattice threshold $r_{l}^{*}$.

\subsection{Harmonic vibrations of the continuum Wigner molecule}

For the third criterion, one needs the zero point vibrational energy of the Wigner molecule that the three particles form when $r_{l}$ is large, but smaller than $r_{l}^{*}$. This can be calculated in the continuum limit, using for $N=3$ the same expansion in powers of $r_{s}^{1 / 2}$ than those used in Eq. [5] for $N \rightarrow \infty$. We summarize the main points, the details being given in Appendix [B] In the continuum, the Hamiltonian $H_{c}$ can written as the sum of two decoupled terms. Denoting $\mathbf{R}=\left(\sum_{i}^{3} \mathbf{r}_{i}\right) / 3$ the coordinate of the center of mass, the first term reads $H_{C M}=\left(\hbar^{2} / 6 m\right) \nabla_{\mathbf{R}}^{2}$ and corresponds to the rigid translation of the molecule while the other term contains the relative motions and the interaction. For a Wigner molecule, the second part can be simplified and expressed in terms of the normal coordinates suitable for describing the small vibrations around equilibrium.

The PRC repulsion is harmonic around equilibrium, and the three particles form a diagonal chain as indicated in the inset of Fig. [4 when $L / 3$ is integer. One gets four decoupled harmonic oscillators, two corresponding to a longitudinal mode of frequency $\omega_{l}=\sqrt{20 B}$, the two others being a transverse mode of frequency $\omega_{t}=\sqrt{8 B}$, where

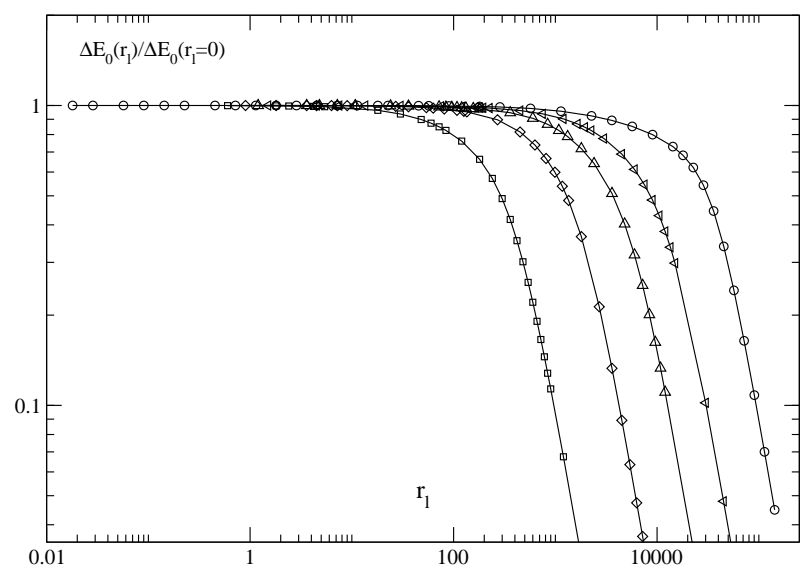

Fig. 6. Dimensionless change $\Delta E_{0}\left(r_{l}\right) / \Delta E_{0}\left(r_{l}=0\right)$ of the GS energy when the longitudinal $\mathrm{BC}$ is twisted for $L=6(\square), 9$ $(\diamond), 12(\triangle), 15(\triangleleft), 18(\circ)$ and $N=3$ as a function of $r_{l}(\mathrm{PRC}$ repulsion).

$B=\left(\sqrt{6} e^{2} \pi\right) /\left(24 D^{3} m\right)$. The zero point vibrational energy is then given by:

$$
\begin{aligned}
E_{v i b}\left(r_{s}, N=3\right) & =\hbar\left(\omega_{l}+\omega_{t}\right) \\
& =2 \pi \frac{\sqrt{5}+\sqrt{2}}{\sqrt{18}}\left(\frac{2}{\pi}\right)^{1 / 4} r_{s}^{-\beta}
\end{aligned}
$$

in rydbergs where $\beta=3 / 2$, with $r_{s}=r_{l} /(2 \sqrt{3 \pi})$ for $N=$ 3 .

\subsection{Scaling of the ground state energy}

From the GS energy $E_{0}(L, U, t)$ of $\mathbf{K}=0$, and for a given value of $N$, we define the dimensionless ratio $F_{N}(L, U, t)$ by:

$$
F_{N}(L, U, t)=\frac{E_{0}(L, U, t)-E_{0}(L, U, t=0)}{E_{0}(L, U=0, t)} .
$$

This ratio gives the change of the GS energy from the Coulomb energy due to the quantum effects, divided by the GS energy without interaction.

The results for the PRC repulsion are shown in Fig. 4 For $t=0$, the values of $L=6,9,12,15,18$ are commensurate with the period of the diagonal Wigner molecule shown in the inset. This gives the same classical Coulomb energy for the lattice and the continuum when $t \rightarrow 0$, eliminating a trivial source of lattice effects. When $F_{N=3}(L, U, t)$ is plotted as a function of $r_{l}$, the different functions $F_{N=3}(L, U, t)$ scale without an observable lattice effect up to the $r_{l}^{*}(L)$ exactly given by Criterion 3 . Using $E_{0}(L, U=0, t)=12 t-8 t-4 t \cos (2 \pi / L)$ one can see that the numerical results coincide with the analytical result $F_{N=3}=0.2327 \sqrt{r_{l}}$ implied by Eq. [35] for intermediate values of $r_{l}$ where one has a continuum Wigner molecule. The function $F_{N=3}(L, U, t)$ saturates to $4 N t / E_{0}(L, U=0, t)$ above $r_{l}^{*}(L)$, as indicated by the dashed lines. 


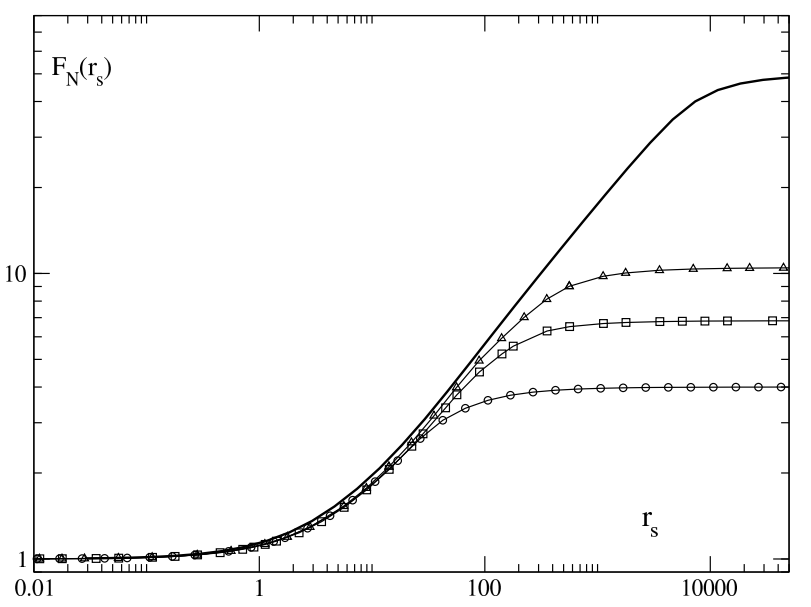

Fig. 7. Energy ratios $F_{N}(L, U, t)$ using the PRC repulsion for $N=3(L=18$ solid line) and $N=4$ with $L=6$ (०), 8 ( $\square), 10$ $(\triangle)$ as a function of $r_{s}=r_{l} /(2 \sqrt{N \pi})$. (PRC repulsion).

\section{Effect on the scaling function when $N$ varies}

In Fig. [7 a small change of the scaling curve $F_{N}\left(r_{s}=\right.$ $r_{l} /(2 \sqrt{N \pi})$ can be seen when a fourth electron is added, accompanied by the expected breakdown of the scaling behavior above the corresponding $r_{l}^{*}$ for $N=4$. When $N \rightarrow \infty, F_{N}$ should converge towards a thermodynamic limit depending only on $r_{s}$. Unfortunately, a study of this convergence is out of reach of a numerical approach using exact diagonalization.

\section{Conclusion}

We have studied the lattice effects upon an interacting system of polarized electrons in two dimensions. We have first considered the case where the number $N$ of polarized electrons is increased in a square lattice of large size $L$ and of fixed parameters $U=e^{2} /\left(\varepsilon_{r} s\right)$ and $t=\hbar^{2} /\left(2 m^{*} s^{2}\right)$. This corresponds to semi-conductor field effect devices or layered oxides where the number of carriers can be varied by an electrostatic gate or by chemical doping. Starting from an empty lattice, one has a continuum regime and its universal scaling if one uses the parameter $r_{s}$, until the carrier density $n_{s}^{*}=1 /\left(\sqrt{\pi} r_{s}^{*} a_{B}^{*}\right)^{2}$ is reached. At this density, the continuum approximation with its universal scaling breaks down, as sketched in the phase diagram given in Fig. 3. This lattice threshold $r_{s}^{*}$ takes place in a quantum fluid phase if the carriers are light and the ratio $s / a_{B}^{*}<23$. We have studied more particularly in the remaining part of our manuscript the case of heavy carriers where $s / a_{B}^{*}>23$, for which the continuum approximation breaks down in the crystalline phase. We have pointed out the limit imposed by the lattice of positive ions upon the zero point motion of the electron lattice. We have neglected the obvious problem coming from the incommensurability of the two lattices, and focus our attention to the commensurate case. The studied lattice effects are independent of this commensurability issue, which should matter at large lattice fillings.

In the second part of this manuscript, we have studied the role of a lattice for a fixed number $N=3,4$ of polarized electrons. The lattice-continuum crossover is then obtained by varying the lattice parameter $r_{l}=(U L) / t$. $r_{l}$ and $r_{s}$ play the same role when $N$ is fixed, since $r_{s}=r_{l} /(2 \sqrt{\pi N})$. The continuum approximation is valid and there is a universal scaling when one uses the parameter $r_{l}$ as far as $r_{l}$ does not exceed a lattice threshold $r_{l}^{*}$ which has been determined from three criteria. One of them was based on the behavior of the persistent current $I\left(r_{l}\right)$ driven by an enclosed Aharonov-Bohm flux. For $r_{l}<r_{l}^{*}(L), I\left(r_{l}\right)=I\left(r_{l}=0\right)$ while $I\left(r_{l}\right)$ decays above $r_{l}^{*}$ and $r_{l}$ ceases to be a scaling parameter. For a finite number of particles, one goes from the continuum regime towards the lattice regime through a smooth crossover. The question to know if this smooth crossover does not become sharper when $N \rightarrow \infty$, to give rise to a true quantum transition is an interesting issue which we postpone to a following study.

Z. Á. Németh acknowledges the financial support provided through the European Community's Human Potential Programme under contract HPRN-CT-2000-00144 and the Hungarian Science Foundation OTKA T034832.

\section{A Weak interaction correction to $r_{s}^{*}$}

When $r_{s}$ is small, the main effect of the interaction is to smear the Fermi surface, giving an uncertainty $\Delta k_{F}$ to $k_{F}$, such that one expects to have the continuum behavior when $k_{F}+\Delta k_{F}$, and not only $k_{F}$, is small. To evaluate $\Delta k_{F}$, we assume that the low excited states only become occupied at low $r_{s}$. The first excitation energy reads:

$$
\begin{aligned}
\Delta E_{1,0} & =t \sum_{i=1}^{N}\left(\mathbf{k}_{i 1}^{2}-\mathbf{k}_{i 0}^{2}\right) \\
& =2 t\left(\mathbf{k}^{\prime 2}-k_{F}^{2}\right) \approx 4 t k_{F} \Delta k
\end{aligned}
$$

where the factor 2 comes from momentum conservation and $k^{\prime}$ is the wave vector of an empty state above the non interacting Fermi surface. This gives us the relation:

$$
\frac{\Delta E_{F}}{\Delta k_{F}} \approx 4 t k_{F}
$$

The Fermi energy uncertainty $\Delta E_{F}$ can be estimated from the spreading $\Gamma$ of a non interacting level, when one turns on the interaction. Using Fermi's golden rule, one gets

$$
\Gamma \approx \frac{2 \pi\left|H_{0,1}\right|^{2} n\left(k_{F}\right)}{\Delta E_{1,0}}
$$

where the matrix element of interaction coupling the GS to the first excited state $\left|H_{0,1}\right|=2 U\left(V(\mathbf{q})-V\left(2 k_{F}\right)\right) \approx$ 
$2 U V(\mathbf{q})$ reads:

$$
H_{01} \approx \frac{U}{L^{2}} \sum_{\mathbf{j}} \frac{e^{i \mathbf{q} \mathbf{j}}}{d_{\mathbf{j} \mathbf{0}}} \approx \frac{U}{L} \int_{0}^{1} \int_{0}^{1} \frac{\cos 2 \pi x}{d(\mathbf{r}, 0)} d^{2} \mathbf{r}=\frac{I U}{L} .
$$

$I$ is a constant equal to 1.029 for the PRC repulsion. The number of states $n\left(k_{F}\right)$ on the Fermi surface is equal to $\sqrt{4 N \pi}$ and $\Delta E_{10}=4 t k_{F}|\mathbf{q}|$ where $|\mathbf{q}|=(2 \pi / L)$ is the smallest momentum for an excitation.

One eventually gets for the Fermi energy uncertainty

$$
\Delta E_{F} \approx \Gamma \approx \frac{U^{2}}{4 t} I^{2}
$$

which gives for the Fermi momentum uncertainty

$$
\Delta k_{F} \approx\left(\frac{U I}{t}\right)^{2} \frac{L}{32 \sqrt{\pi N}} .
$$

The condition

$$
k_{F}+\Delta k_{F}<\frac{\pi}{2}
$$

is satisfied if

$$
r_{s}-\frac{I^{2}}{16} r_{s}^{3}>\frac{4}{\pi} \frac{s}{a_{B}}
$$

When $r_{s}$ is not too large, the continuum theory is valid if $r_{s}>r_{s}^{*}$ with a threshold $r_{s}^{*}$ having a small correction $\propto\left(s / a_{B}\right)^{3}$ driven by the interaction:

$$
r_{s}^{*} \approx \frac{4}{\pi} \frac{s}{a_{B}}+\frac{4 I^{2}}{\pi^{3}}\left(\frac{s}{a_{B}}\right)^{3} .
$$

The constants in the expression of $r_{s}^{*}$ depend on the used criterions for neglecting lattice effects (for instance $4 / \pi$ comes from the condition (43)).

\section{B Zero point energy of a continuum Wigner molecule for $N=3$}

For three spinless fermions on a continuum square domain of size $D$ with periodic BCs, the continuum PRC repulsion reads

$$
V(\mathbf{r})=\frac{e^{2} \pi}{D \sqrt{\sin ^{2} \frac{r_{x} \pi}{D}+\sin ^{2} \frac{r_{y} \pi}{D}}} .
$$

If $D$ is large enough, the GS is a "Wigner molecule" of delocalized center of mass, but of quasi-localized interparticle spacings for minimizing the Coulomb energy. For a certain center of mass, the molecule of lowest Coulomb energy with the repulsion (46) consists in putting the particle coordinates at $\mathbf{r}_{1}=(0,0), \mathbf{r}_{2}=(D / 3, D / 3)$ and $\mathbf{r}_{3}=(-D / 3,-D / 3)$. This configuration has the Coulomb energy

$$
E_{C o u l}=\sqrt{6} e^{2} \pi / D .
$$

The particles forming this molecule vibrate around the equilibrium positions. This motion is an harmonic oscillation if the amplitude of the vibration is small. To describe this harmonic motion, one expands the pair-potential (46) around the equilibrium distance $\mathbf{r}_{0}=(D / 3, D / 3)$ up to the second order:

$$
\begin{aligned}
V(\mathbf{r}) & \approx \frac{\sqrt{6} e^{2} \pi}{3 D}+(\ldots) \\
& +\frac{7 \sqrt{6}}{72} \frac{e^{2} \pi}{D^{3}}\left(\left(x-\frac{D}{3}\right)^{2}+\left(y-\frac{D}{3}\right)^{2}\right) \\
& +\frac{\sqrt{6}}{12} \frac{e^{2} \pi}{D^{3}}\left(r_{x}-\frac{D}{3}\right)\left(r_{y}-\frac{D}{3}\right)+O\left(r^{3}\right),
\end{aligned}
$$

where the missing term $(.$.$) is the first order contribution$ which disappears after summing over all the pair potentials. The expansion (48) becomes:

$$
V(\mathbf{r}) \approx \frac{\sqrt{6} e^{2} \pi}{3 D}+(\ldots)+\left(\mathbf{r}-\mathbf{r}_{0}\right)\left(\begin{array}{cc}
A & B \\
B & A
\end{array}\right)\left(\mathbf{r}-\mathbf{r}_{0}\right)
$$

where

$$
A=\frac{7 \sqrt{6}}{72} \frac{e^{2} \pi}{D^{3}}
$$

and $B=3 A / 7$.

The three particle Hamiltonian with the expanded repulsion becomes $H_{c} \approx E_{\text {Coul }}+H_{\text {harm }}$, where the harmonic part is:

$$
H_{h a r m}=-\frac{\hbar^{2}}{2 m}\left(\nabla_{1}^{2}+\nabla_{2}^{2}+\nabla_{3}^{2}\right)+\boldsymbol{X} \hat{M} \boldsymbol{X} .
$$

The vector $\boldsymbol{X}=\left(x_{1}, y_{1}, x_{2}, y_{2}, x_{3}, y_{3}\right)$ is composed of the 6 relative coordinates and the $6 \times 6$ matrix $\hat{M}$ is given by:

$$
\hat{M}=\left(\begin{array}{cccccc}
2 A & 2 B & -A & -B & -A & -B \\
2 B & 2 A & -B & -A & -B & -A \\
-A & -B & 2 A & 2 B & -A & -B \\
-B & -A & 2 B & 2 A & -B & -A \\
-A & -B & -A & -B & 2 A & 2 B \\
-B & -A & -B & -A & 2 B & 2 A
\end{array}\right)
$$

Diagonalizing $\hat{M}$, one obtains the normal modes of the harmonic oscillations while the eigenvalues of $\hat{M}$ give their frequencies. One obtains

- Two eigenvectors of eigenvalue 0 .

$$
\begin{aligned}
& \chi_{1}=\frac{1}{\sqrt{3}}(1,0,1,0,1,0) \cdot \boldsymbol{X} \\
& \chi_{2}=\frac{1}{\sqrt{3}}(0,1,0,1,0,1) \cdot \boldsymbol{X} .
\end{aligned}
$$

This zero frequency mode corresponds to the translation of the center of mass of the molecule.

- Two other eigenvectors of eigenvalue $10 B$, corresponding to the longitudinal mode (vibration parallel to the axis of the molecule). The normal coordinates can be taken as:

$$
\begin{aligned}
& \chi_{3}=\frac{1}{2}(1,1,-1,-1,0,0) \cdot \boldsymbol{X} \\
& \chi_{4}=\frac{1}{\sqrt{12}}(1,1,1,1,-2,-2) \cdot \boldsymbol{X}
\end{aligned}
$$


- Two eigenvectors of eigenvalue $4 B$, corresponding to the transverse modes. The normal coordinates can be taken as:

$$
\begin{aligned}
& \chi_{5}=\frac{1}{2}(1,-1,-1,1,0,0) \cdot \boldsymbol{X} \\
& \chi_{6}=\frac{1}{\sqrt{12}}(1,-1,1,-1,-2,2) \cdot \boldsymbol{X}
\end{aligned}
$$

Using these normal coordinates, the Hamiltonian (51) becomes a decoupled sum of two harmonic oscillators:

$H_{h a r m}=-\frac{\hbar^{2}}{2 m} \sum_{\alpha=1}^{6} \frac{\partial^{2}}{\partial \chi_{\alpha}^{2}}+10 B\left(\chi_{3}^{2}+\chi_{4}^{2}\right)+4 B\left(\chi_{5}^{2}+\chi_{6}^{2}\right)$

For a GS of total momentum $\mathbf{K}=0$, there is no motion of the center of mass, the GS wave-function does not depend on $\chi_{1}$ and $\chi_{2}$ and can be factorized as:

$$
\Psi\left(\chi_{1}, \ldots, \chi_{6}\right)=\varphi_{0 L}\left(\chi_{3}\right) \varphi_{0 L}\left(\chi_{4}\right) \varphi_{0 T}\left(\chi_{5}\right) \varphi_{0 T}\left(\chi_{6}\right)
$$

where $L, T$ refers to the transverse and longitudinal modes and $\varphi_{0}$ to the ground state of an harmonic oscillator:

$$
\varphi_{0}(x)=\frac{1}{l_{\omega}^{1 / 2} \pi^{1 / 4}} \exp -\frac{x^{2}}{2 l_{\omega}^{2}}
$$

of length $l_{\omega}=\left(\hbar^{2} /\left(m^{2} \omega^{2}\right)\right)^{1 / 4}$. One eventually obtains for the GS energy with the expanded pair potentials:

$$
\begin{aligned}
E_{0}-E_{\text {Coul }} & =\hbar\left(\omega_{T}+\omega_{L}\right) ; \\
\omega_{L} & =\sqrt{\frac{20 B}{m}}, \\
\omega_{T} & =\sqrt{\frac{8 B}{m}}
\end{aligned}
$$

and using the expression of $B$ :

$$
\begin{aligned}
E_{0}-E_{\text {Coul }} & =\sqrt{\frac{20 \sqrt{6}}{24} \frac{\hbar^{2} e^{2} \pi^{3}}{D^{3} m}}+\sqrt{\frac{8 \sqrt{6}}{24} \frac{\hbar^{2} e^{2} \pi^{3}}{D^{3} m}} \\
& =(\sqrt{5}+\sqrt{2}) \sqrt{\frac{\sqrt{6} \pi^{3}}{3}} \sqrt{\frac{U t}{L^{3}}}
\end{aligned}
$$

For the energy ratio $F_{N=3}(L, U, t)$, using for the kinetic energy in the continuum limit $E_{0}(L, U=0, t)=8 \pi^{2} t / L^{2}$, one gets the behavior numerically obtained from the lattice Hamiltonian $H_{l}$ and shown in Fig. 4 for intermediate $r_{s}$ :

$$
\begin{aligned}
F_{0}\left(r_{s}\right) & =\frac{\sqrt{5}+\sqrt{2}}{8 \pi^{2}} \sqrt{\frac{\sqrt{6} \pi^{3}}{3}} \sqrt{\frac{U L}{t}} \\
& =\frac{\sqrt{5}+\sqrt{2}}{\sqrt{96}}\left(\frac{18}{\pi}\right)^{1 / 4} \sqrt{r_{s}} \\
& =0.5764 \sqrt{r_{s}}=0.2327 \sqrt{r_{l}}
\end{aligned}
$$

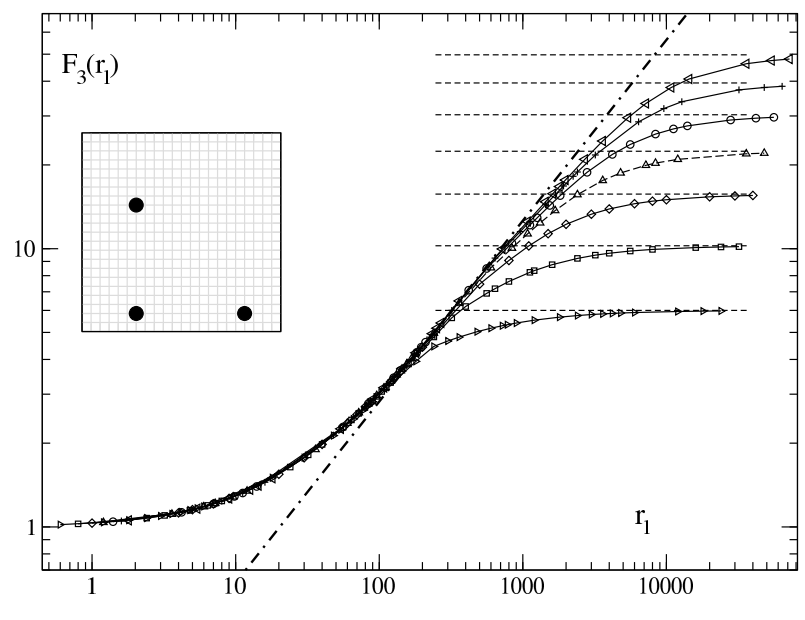

Fig. 8. Energy ratio $F_{N=3}(L, U, t)$ as a function of $r_{l}=U L / t$ using the PSC repulsion for $L=6(\triangleright), 8(\square), 10(\diamond), 12(\triangle), 14$ $(\circ), 16(+), 18(\triangleleft)$. The dotted-dashed line gives the $r_{l}^{2 / 3}$ behavior due to the vibrations of the continuum Wigner molecule. Inset: a GS configuration when $t=0$ and $L=24$.

\section{Lattice threshold $r_{l}^{*}$ using the PSC repulsion}

With the distance $d_{\mathbf{j} \mathbf{j}^{\prime}}$ defined by Eq. 31, we have previously studied the validity of a continuum approximation for a lattice model of three polarized electrons. In this appendix, we revisit the same issue defining $d_{\mathbf{j} \mathbf{j}^{\prime}}$ from Eq. [30] instead of Eq. 31] Let us calculate the quantities used for the three criteria when one uses the PSC repulsion. For $t=0$, the Wigner "molecule" minimizing the PSC Coulomb energy has the triangular shape shown in the inset of Fig. 8 instead of the linear shape shown in the inset of Fig. 4 Moving one particle by a single hop in this triangular molecule increases the PSC Coulomb energy by an amount

$$
\Delta E_{\text {Coul }}^{(P S C)} \approx \frac{\sqrt{2} U}{L^{2}}
$$

when $L$ is sufficiently large, instead of the $\Delta E_{\text {Coul }}^{(P R C)} \propto$ $U / L^{3}$ given by Eq. 32

For the energy change $\Delta E_{0}$, one obtains the same expressions as in Eq. 33. but with $\Delta E_{C o u l}$ given by the Eq. 64 instead of Eq. 32 The two first criteria gives

$$
r_{l}^{*}(L)=A L^{\alpha}
$$

where $\alpha=3$ for the PSC repulsion, instead of $\alpha=4$ for the PRC repulsion.

When one takes the PSC repulsion, the three relative distances at equilibrium are precisely $\mathbf{r}=(L / 2, L / 2), \mathbf{r}=$ $(0, L / 2)$ and $\mathbf{r}=(L / 2,0)$ respectively when $L$ is even. The potentials $v(\delta \mathbf{r})$ felt by the electrons around their equilibrium positions are singular and instead of the analytical expansion (48) of $v(\delta \mathbf{r})$, one has $v(\delta \mathbf{r}) \approx C_{1}\left|\delta r_{x}\right|+C_{2}\left|\delta r_{y}\right|$, where $C_{1}$ and $C_{2}$ depend on the equilibrium positions 
and are $\propto e^{2} / D^{2}=U / L^{2}$. For a single particle in a $1 d$ potential $v(x)=C|x|$, the GS energy $\epsilon$ can be approximated by $t / B^{2}+C B$ where $B$ is the GS extension and is given by $\partial \epsilon / \partial B=0$. This yields $B \propto(C / t)^{1 / 3}$ and $\epsilon \propto\left(U^{2} t / L^{4}\right)^{1 / 3}$. Since the $2 d$-potential $v(\delta \mathbf{r})$ is separable, one eventually finds:

$$
E_{v i b}^{(P S C)}\left(r_{s}, N=3\right) \propto r_{s}^{-\beta}
$$

in rydbergs where $\beta=4 / 3$. As one can see, the PSC repulsion gives a higher exponent $\beta$ when $N=3$, which is inconsistent with the usual expansion [2] in powers of $r_{s}^{-1 / 2}$ first proposed by Wigner.

Using Eq. 66] one gets from Criterion $3 r_{l}^{*}$ given by Eq. 34 again, but with $\alpha=3$ for the PSC repulsion instead of $\alpha=4$ for the PRC repulsion. The PSC repulsion is somewhat unphysical and leads to stronger lattice effects, but provides an interesting check of the validity of our theory: The changes of $\Delta E_{\text {Coul }}^{(P S C)}$ and $E_{v i b}^{(P S C)}$ are such that the different criterions give thresholds $r_{l}^{*}$ which are consistent.

The dimensionless energy ratio $F_{N}(L, U, t)$ for the PSC repulsion is shown in Fig. 8 for even values of $L$, where the GS is a triangular "molecule" shown in the inset when $t / U \rightarrow 0$. Again the curves scale up to the onset $r_{l}^{*}(L)$ given by Criterion 3 . But the PSC repulsion gives rise to a different onset $r_{l}^{*}(L)$ than the PRC repulsion for $N=3$, since at intermediate $r_{l}$ one has $F_{N=3} \propto r_{l}^{2 / 3}$ for the PSC repulsion, and not $\propto r_{l}^{1 / 2}$ as for the PRC repulsion.

Does this difference remain for larger values of $N$ ? Indeed the contribution of pairs $i j$ having the coordinates of their spacings $d_{i j}$ close to $D / 2$, and responsible for the $r_{s}^{2 / 3}$ behavior when $d_{i j}$ is defined by Eq. [30] becomes a surface effect $\propto N$ compared to the bulk contribution $\propto N^{2}$ of the remaining pairs, yielding $\Delta E_{\text {Coul }}^{P S C} \approx A N / L^{2}+B N^{2} / L^{3}$, where $A$ and $B$ are constant. For a fixed $L$ and increasing $N, \Delta E_{\text {Coul }}^{P S C} \rightarrow B N^{2} / L^{3}$ and following Criterion 1, the conventional $r_{s}^{1 / 2}$ expansion for $F_{N}$ should be valid for the PSC repulsion too. Therefore, large periodic square lattices should exhibit a behavior independent of the choice of the long range part of the Coulomb repulsion when $N$ becomes large. Another possible choice is the Ewald repulsion obtained after summing over all the electrons present in the infinite repetition of the same finite square in the $x$ and $y$ directions. For a small number $N$ of electrons in a periodic square, these definitions are somewhat arbitrary. But to reach the thermodynamic limit, the PSC repulsion is less appropriate than the PRC or Ewald repulsions, since it gives larger finite $N$ effects.

Nevertheless, the following relations for the lattice threshold, the continuum zero point energy of the crystalline oscillation and the characteristic scale of the Coulomb energy respectively:

$$
\begin{aligned}
r_{l}^{*} & \propto L^{\alpha} \\
E_{\text {vib }} & \propto r_{l}^{-\beta} \\
\Delta E_{\text {Coul }} & \propto U L^{-\gamma}
\end{aligned}
$$

remain valid independently of the used definition of the Coulomb repulsion in the periodic square lattice,

$$
\alpha=\gamma+1, \alpha=\frac{2}{2-\beta}
$$

between the exponents.

\section{References}

1. M. Gell-Mann and K. A. Brueckner, Phys. Rev. 106, 364 (1957).

2. E. Wigner, Trans. Faraday Soc. 34, 678 (1938).

3. W. J. Carr, Phys. Rev. 122, 1437 (1961).

4. L. Bonsall and A. A. Maradudin, Phys. Rev. 15, 1959 (1977).

5. D.M. Ceperley, Phys. Rev. B 18, 3126 (1978).

6. B. Tanatar and D.M. Ceperley, Phys. Rev. B 39, 5005 (1989).

7. J. González, Phys. Rev. B 63, 024502 (2000).

8. J. González, F. Guinea and M.A.H. Vozmediano, Europhys. Lett. 34, 711 (1996)

9. D. Zanchi and H.J. Schulz, Phys. Rev. B 54, 9509 (1996).

10. D. Weinmann, J.-L. Pichard and Y. Imry, J. Phys. I France 7, 1559 (1997).

11. G. Katomeris, F. Selva and J.-L. Pichard, Eur. Phys. J. B 31401 (2003).

12. Z. Á. Németh and J.-L. Pichard, Eur. Phys. J. B 338799 (2003).

13. M. Martínez and J.-L. Pichard, Eur. Phys. J. B 3093 (2002).

14. F. Selva and D. Weinmann, Eur. Phys. J. B 18137 (2000).

15. J. Ruvalds, C. T. Rieck, S. Tewari J. Thoma and A. Virosztek, Phys. Rev. B 51, 3797 (1995).

16. G. G. Amatucci, J. M. Tarascon and L. C. Klein, J. Electrochem. Soc 143, 1114 (1996); K. Takada et al, Nature 42253 (2003).

17. M. Roger and N. Shannon, unpublished.

18. P.W. Anderson, Phys. Rev. 109, 1492 (1958).

19. D. Weinmann and J.-L. Pichard, Phys. Rev. Lett. 77, 1556 (1996).

20. D. L. Shepelyansky and O. P. Sushkov, Europhys. Lett. 37, 121 (1997).

21. G. Benenti, G. Casati and D. L. Shepelyansky, Eur. Phys. J. D 17265 (2001).

22. B. L. Altshuler, Y. Gefen, A. Kamenev and L. Levitov, Phys. Rev. Lett. 78, 2803 (1997).

23. P. Jacquod and D. L. Shepelyansky, Phys. Rev. Lett. 79, 1837 (1997).

24. A. Müller-Groeling and H. A. Weidenmüller, Phys. Rev. B 49, 4752 (1994).

25. I. V. Krive, P. Sandström, R. I. Shekhter, S. M. Girvin and M. Jonson, Phys. Rev. B 52, 16451 (1995).

26. G. Burmeister and K. Maschke, Phys. Rev. B 65, 155333 (2002). 